\title{
Turbulence Regeneration in Pipe Flow at Moderate Reynolds Numbers
}

\author{
Björn Hof, ${ }^{*}$ Casimir W. H. van Doorne, Jerry Westerweel, ${ }^{\dagger}$ and Frans T. M. Nieuwstadt ${ }^{\ddagger}$ \\ Laboratory for Aero- and Hydrodynamics, Delft University of Technology, Leeghwaterstraat 21, 2628 CA Delft, The Netherlands
}

(Received 20 May 2005; published 17 November 2005)

\begin{abstract}
We present the results of an experimental investigation into the nature and structure of turbulent pipe flow at moderate Reynolds numbers. A turbulence regeneration mechanism is identified which sustains a symmetric traveling wave within the flow. The periodicity of the mechanism allows comparison to the wavelength of numerically observed exact traveling wave solutions and close agreement is found. The advection speed of the upstream turbulence laminar interface in the experimental flow is observed to form a lower bound on the phase velocities of the exact traveling wave solutions. Overall our observations suggest that the dynamics of the turbulent flow at moderate Reynolds numbers are governed by unstable nonlinear traveling waves.
\end{abstract}

DOI: 10.1103/PhysRevLett.95.214502

PACS numbers: 47.27.Cn

Turbulent shear flows have been a major focus of fluid dynamics research since the classical experiments of Reynolds [1]. Flows such as that of a fluid down a straight pipe are known to become turbulent at large enough flow rates. Stability theory, on the other hand, suggests [2] that laminar pipe flow is stable to infinitesimal perturbations for all values of the nondimensionalized flow rate, the Reynolds number $\operatorname{Re}$. (Here $\operatorname{Re}=U D / \nu$, where $U$ is the mean velocity, $D$ is the diameter of the pipe, and $\nu$ is the kinematic viscosity of the fluid.) Similar observations have been made in related shear flows such as Couette [3-5], Poiseuille, and boundary layer flows where turbulence sets in despite linear stability [2] or the linear instability mechanism can be bypassed. Understanding the transition scenario and the nature of the resulting turbulent flows is one of the greatest challenges in fluid dynamics.

A process which is believed to be of relevance for transition in shear flows is the so called lift-up mechanism [6]. Here streamwise vortices of relatively small magnitude transport low momentum fluid away from the wall and high momentum fluid towards the wall creating strong anomalies in the velocity profile, so-called low- and high-speed streaks (elongated regions of low or high streamwise velocity with respect to their surroundings). During this process the initial perturbation amplitude can grow substantially. Since the mechanism is of a linear nature, in linearly stable flows eventually all perturbations will decay. However it is assumed that once the perturbation has reached a sufficiently large amplitude nonlinear effects will become important and lead to secondary instabilities. The mathematical reason for the transient growth of initial perturbations is the non-normality of the linearized NavierStokes operator and this mechanism has attracted considerable attention [7-9].

In more recent studies the relevance of nonlinear effects and, in particular, the role of nonlinear solutions has been discussed $[10,11]$. Such solutions have been calculated for Couette and Poiseuille flow [12-16] and more recently also for pipe flow $[17,18]$. These new flow states arise at finite values of the control parameter (Re) and in the case of pipe flow they take the form of traveling waves (TWs), which are coherent flow structures propagating at a constant phase speed in the streamwise direction. Typically these TW solutions are unstable so that the laminar flow state remains the only stable solution. In analogy to observations in low dimensional models $[19,20]$ it has been suggested that as the Reynolds number is increased further the unstable states undergo secondary bifurcations and form an attracting region in phase space which gives rise to turbulent dynamics. The basin of attraction of the turbulent state grows with increasing Reynolds number, so that smaller and smaller perturbations are sufficient to destabilize the laminar flow.

Confirmation of this picture has been provided by recent experiments in pipe flow where transients of unstable TWs have been observed that are in close agreement with the numerical solutions [21]. Further experimental investigations have demonstrated that the stability boundary between the laminar and the turbulent flow indeed decreases [22-24], which supports the view of a diminishing basin of attraction of the laminar state.

A self-regeneration cycle for such TW and periodic solutions has been proposed by Hamilton et al. and Waleffe [25,26]. In the initial step, streamwise vortices create streaks essentially through the lift-up mechanism discussed above. This configuration of low- and highspeed streaks is inflexionally unstable, leading to a wavy modulation of the streaks in the streamwise direction. Finally, the nonlinear development of the streak instability regenerates streamwise vortices, closing the feedback loop. This self-sustaining process (SSP) was first observed by constraining a turbulent flow to a small computational box with periodic boundary conditions [25] which resulted in the spontaneous formation of the above described three-dimensional vortex streak configuration. Similarly in a numerical study of turbulence in a minimal flow unit [27,28] a TW consisting of a wavy streak aligned by streamwise vortices was identified in a wall shear flow. Wavy streaks sandwiched between counterrotating streamwise vortices have also been observed by 
Brandt et al. [29] during the transition process in boundary layers.

In this Letter we present the first experimental observation of the self-regeneration cycle in a turbulent shear flow. Whereas all previous observations of this cycle were made in numerical confinements with periodic boundary conditions, in our study no periodicity is imposed on the system. In additional measurements we relate the advection speed of macroscopic turbulent structures such as turbulent puffs and slugs to the phase speed of TWs.

A detailed description of the experimental setup can be found in $[21,30]$. The measurements were carried out in a $26 \mathrm{~m}$ long pipe made of $2 \mathrm{~m}$ long perspex sections with an inner diameter of $4 \mathrm{~cm}$. The working fluid was water and the flow was driven by a pump. A smooth contraction in combination with several screens and meshes at the pipe entrance ensured that the flow could be kept laminar up to $\operatorname{Re} \approx 60000$ [23]. The laminar flow was perturbed 450 pipe diameters downstream of the inlet, where the laminar profile was fully developed. The perturbation consisted of a water jet which was injected perpendicularly to the mean flow through a $1 \mathrm{~mm}$ hole in the pipe wall. Velocity and duration of the jet were controlled by a syringe pump. Typically amplitudes were chosen sufficiently large to cause transition to turbulence. The socreated turbulent region was investigated a further 150 pipe diameters downstream with a stereoscopic highspeed particle image velocimetry (PIV) system. Here a cross-sectional plane of the pipe is illuminated with a laser light sheet using a pulsed Nd:YLF laser and this plane is viewed with two $1000 \mathrm{~Hz}$ cameras positioned in forward scatter on opposite sides of the light sheet at $45^{\circ}$ to the observation plane. For visualization purposes the water was seeded with $10 \mu \mathrm{m}$ spherical particles. During a single PIV measurement each camera records two images $3 \mathrm{~ms}$ apart. The displacement of the tracer particles between the two recorded images allows the reconstruction of the inplane velocity field. The additional information gained from the second camera angle enables us to also determine the out-of-plane component so that the full three component velocity field in the measurement plane is obtained [31]. In the first measurement reported here a turbulent region at $\mathrm{Re}=3000$ is investigated. The turbulent structures found in this Reynolds number regime are called turbulent slugs [32]. They travel in the downstream direction at approximately the bulk flow speed, which is $7 \mathrm{~cm} / \mathrm{s}$. The sampling rate of the PIV system was set to $250 \mathrm{~Hz}$. Hence approximately 140 independent PIV measurements were recorded while the turbulent slug was advected by a nondimensional distance of 1 pipe diameter in the streamwise direction. Since the advection speed of the turbulent region is approximately 10 times larger than the crossstream components, changes in the velocity field due to the turbulent motion are small over the observation time (approximately $2 \mathrm{~s}$ ). Consequently, the time spacing of the measurements can be converted into a spatial separation in the mean flow direction (Taylor's hypothesis) allowing the full spatial reconstruction of the turbulent structure. Four velocity fields in the cross-sectional plane measured during the passage of the turbulent slug are shown in Fig. 1. The colors indicate the relative streamwise velocity (see figure caption for details) and high- and low-speed streaks are shown in red (dark gray) and blue (gray). The arrows depict the cross-stream velocities. The four cross sections shown in Fig. 1 are separated by $0.1 \mathrm{~s}$, which, based on the bulk flow speed, corresponds to a spacing of 0.18 pipe diameters. Four elongated high-speed streaks can be observed close to the pipe wall and a low-speed streak region dominates the central part of the pipe. The streak configuration is approximately symmetric to a rotation by $180^{\circ}$ with respect to the central point. The rotational symmetry of the streak structure in the cross-sectional plane and the dynamics of the low-speed streaks in the streamwise direction are characteristic for a TW. Two distinct low-speed streak arms are distinguishable, which initially point outwards from the pipe center in the horizontal direction. They subsequently undergo an antisymmetric up and down motion while the high-speed streaks essentially remain in the same position. This dynamical behavior is in excellent qualitative agreement with that reported for the numerically observed TWs $[17,18]$. The reconstruction of the three-dimensional streak structure is shown in Fig. 2. Again high-speed streaks are shown in red and low-speed streaks in blue and the periodic modulation of the lowspeed structure corresponding to the up and down motion of the left streak in Fig. 1 is clearly observable here. As

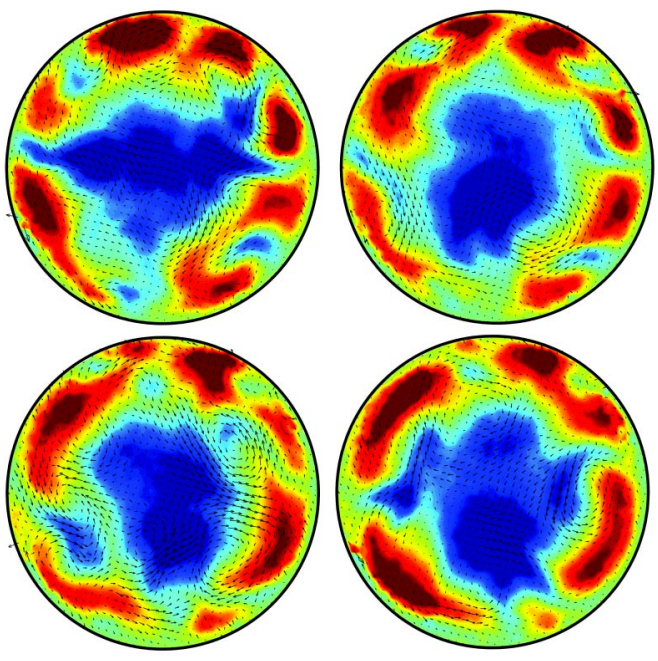

FIG. 1 (color online). Traveling wave transient observed in a turbulent slug at $\mathrm{Re}=3000$. The frames are separated by $0.2 \mathrm{~s}$, which corresponds to a spatial separation of $0.18 \mathrm{D}$. The color scheme represents the streamwise velocity component. Instead of subtracting the laminar profile (LP) from the streamwise component as in previous studies $[17,21]$ we have here chosen to subtract $0.5 \mathrm{LP}+0.5 \mathrm{TP}$, where $\mathrm{TP}$ is the time averaged turbulent profile at $\mathrm{Re}=3000$. We found that this visualization method resolves the streaks in the near wall region more clearly than subtraction of the laminar profile alone. The cross-stream components are indicated by the arrows. 
discussed above, the streamwise periodic modulation manifests the wavy instability of the streak, which is an essential part of the SSP. In Fig. 3 the same streak is displayed together with the streamwise vorticity. As suggested in the turbulence regeneration cycle, the streak is sandwiched between two counter-rotating streamwise vortices, which are shown in yellow and red. A second pair of streamwise vortices aligns the streak approximately $0.7 \mathrm{D}$ further downstream. The periodicity of the vortices confirms the regeneration of the streamwise vorticity by the wavy instability of the streak and closes the feedback loop of the SSP. Overall, Fig. 3 is in very close agreement with the streak instabilities observed in the numerical studies $[14,28]$. From the periodic modulation of the streak and the streamwise vortices the wavelength of the TW can be estimated to approximately $0.75 \mathrm{D}$. This value is well within the bandwidth of wavelength observed for twofold or fourfold waves in the numerical studies [17,18].

A further test of the relevance of TWs to turbulent shear flows can be sought in a comparison of the phase speed of such TWs to the typical advection speeds observed for macroscopic turbulent structures. Depending on the Reynolds number two different turbulent structures are found in transitional pipe flow, turbulent slugs and puffs [32]. The former occur at $\operatorname{Re}>2600$ and the latter are observed for $\operatorname{Re}<2600$. The main difference between the two is that slugs have a sharp laminar turbulence interface at their leading edge and at their trailing edge; for puffs only the trailing interface is sharp. Here we concentrate on

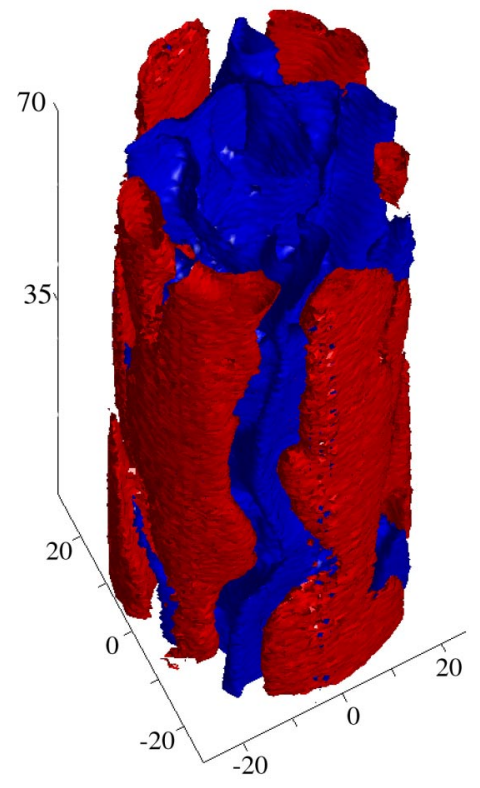

FIG. 2 (color online). Reconstruction of the three-dimensional streak structure of the traveling wave. High and low-speed streaks are shown in red and blue, length units are in milimeters. The blue low-speed streak undergoes a wavy instability. The overall three-dimensional structure is in excellent agreement with that of similar traveling wave solutions observed in numerical simulations [18]. the advection speed of the trailing edge (i.e., the upstream laminar turbulence interface), which is the slowest part of these turbulent structures. Whereas in previous studies it has been reported that the trailing edge travels at velocities slower than the mean velocity [32], the phase speeds reported for TW solutions are typically larger than the mean velocity. Our measurements of the trailing edge velocity are represented by the square and circular symbols in Fig. 4, whereas the triangles correspond to the data of previous experimental studies [32]. In our measurements the experimental procedure was again to create localized regions of turbulence by injection of a jet. Subsequently, the center line velocity was monitored with a laser doppler anemometer several hundred pipe diameters downstream. Each data point represents the average over 10 realizations of a turbulent puff or slug. The advection velocities were determined from the time that passed between the perturbation and the passage of the trailing edge of the puff at the measurement point. The distance between the perturbation and the measurement location was $300 \mathrm{D}$ in the experiments presented by the (green) circles and $150 \mathrm{D}$ for these presented by the (red) squares. For $\operatorname{Re}>2000$ both data sets show very close agreement indicating that the trailing edge travels at a constant speed downstream. The data are also in good agreement with the previous measurements [32] presented by the (blue) triangles in Fig. 4. Whereas previous studies did not extend below $\operatorname{Re}=2000$, here we find that the advection speeds of the turbulent puffs indeed becomes larger than the bulk velocities for $\operatorname{Re}<2000$. Below Re, 1800 turbulence is not sustained but can only

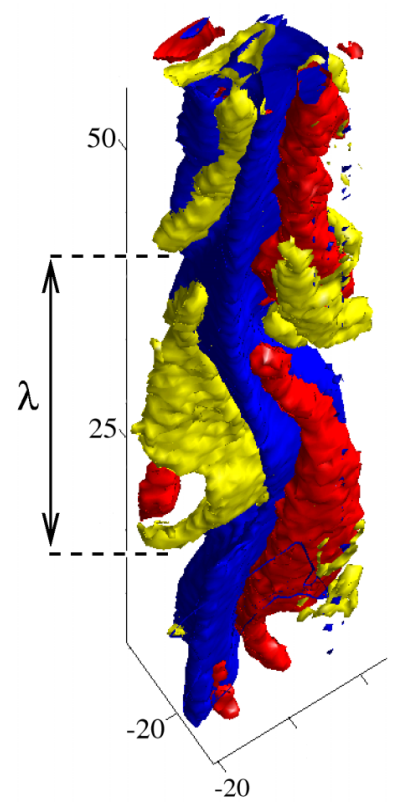

FIG. 3 (color online). Three-dimensional reconstruction of the wavy low-speed streak and the streamwise vorticity. The wavy streak (blue) is sandwiched between counter-rotating streamwise vortices (yellow and red). Positive and negative vorticity is shown in yellow and red. The periodicity of the wave is evident from the wavy streak as well as from the vorticity field. 


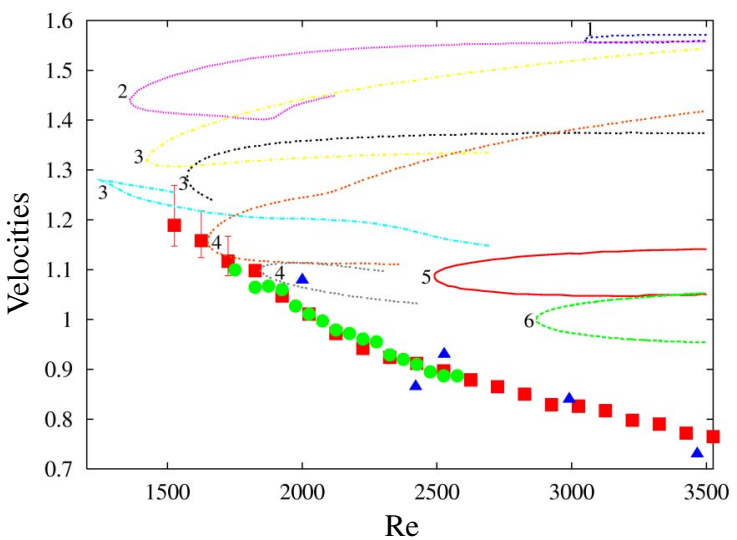

FIG. 4 (color online). The data points give the speed of the trailing edge of turbulent puffs and slugs as a function of Re (see text for details). The curves show the phase speed of the traveling wave solutions at their optimal wavelength as observed by Wedin and Kerswell [18]. The numbers denote the $n$-fold rotational symmetry of the respective TWs. Velocities are normalized by the bulk/mean velocity.

be observed in transients. In agreement with numerical studies [19] we observed that the lifetimes of these transients (i.e., the distance they travel downstream before they decay) decreases with decreasing Re. We also observed that the propagation of these transients increases towards the end of their lifetime. We have marked the propagation speeds measured for the three lowest Reynolds numbers investigated with error bars. Here the actual data point indicates the measured mean velocity over a distance of $62 \mathrm{D}$, the lower end of the error bar shows the speed measured over the first $37 \mathrm{D}$ and the upper end the speed over the last $25 \mathrm{D}$. The curves superimposed on the data show the phase speeds of the traveling waves observed numerically [18]. Especially at the lower Reynolds numbers, the data points accurately mark the line of minimum phase speeds of the TWs at their optimal wavelength. Overall the speed of the trailing edge of puffs and slugs forms a lower bound for the phase speeds of the TWs observed so far. This observation is in line with the suggestion that unstable TWs provide the building blocks for these turbulent flow structures. Further experimental and numerical studies are necessary to clarify if turbulent puffs and slugs can be interpreted as wave packages of nonlinear TWs. Since it is likely that many other TW solutions exist it will be interesting to observe if their phase speeds will also lie above the limit set by the advection speeds of puffs and slugs.

In conclusion we observed a turbulence sustaining mechanism which has previously only been identified in numerical calculations and which is believed to be of relevance to transition [29] and energy regeneration in shear flows $[14,25,28]$. This mechanism was identified as part of a traveling wave transient in the turbulent flow. Our measurement of the advection speed of turbulent puffs suggests that the speed of the upstream laminar turbulence interface is related to the phase speed of the slowest relevant TW. Overall our observations support the proposition that turbulent pipe flow is organized around unstable nonlinear traveling waves.

*Present address: Nonlinear and Liquid Crystal Physics Group, School of Physics and Astronomy, Brunswick Road, University of Manchester, Manchester M13 9PL, United Kingdom.

Electronic address: bjorn@ @eynolds.ph.man.ac.uk †Electronic address: J.Westerweel@wbmt.tudelft.nl ${ }^{*}$ Deceased.

[1] O. Reynolds, Philos. Trans. R. Soc. London 174, 935 (1883).

[2] P. Drazin and W. Reid, Hydrodynamic Stability (Cambridge University Press, Cambridge, England, 1981).

[3] N. Tillmark and P. H. Alfredsson, J. Fluid Mech. 235, 89 (1992).

[4] F. Daviaud, J. Hegseth, and P. Bergé, Phys. Rev. Lett. 69, 2511 (1992).

[5] O. Dauchot and F. Daviaud, Phys. Fluids 7, 335 (1995).

[6] M. Landahl, J. Fluid Mech. 98, 243 (1980).

[7] L. Boberg and U. Brosa, Z. Naturforsch., A: Phys. Sci. 43, 697 (1988).

[8] L. N. Trefethen, A.E. Trefethen, S.C. Reddy, and T. Driscol, Science 261, 578 (1993).

[9] S. Grossmann, Rev. Mod. Phys. 72, 603 (2000).

[10] O. Dauchot and P. Manneville, J. Phys. II (France) 7, 371 (1997).

[11] F. Waleffe, Phys. Fluids 7, 3060 (1995).

[12] M. Nagata, J. Fluid Mech. 217, 519 (1990).

[13] R. Clever and F. Busse, J. Fluid Mech. 344, 137 (1997).

[14] F. Waleffe, Phys. Rev. Lett. 81, 4140 (1998).

[15] F. Waleffe, Phys. Fluids 15, 1517 (2003).

[16] F. Waleffe, J. Fluid. Mech. 435, 93 (2001).

[17] H. Faisst and B. Eckhardt, Phys. Rev. Lett. 91, 224502 (2003).

[18] H. Wedin and R. Kerswell, J. Fluid Mech. 508, 333 (2004).

[19] H. Faisst and B. Eckhardt, J. Fluid Mech. 504, 343 (2004).

[20] B. Eckhardt and A. Mersmann, Phys. Rev. E 60, 509 (1999).

[21] B. Hof, C. van Doorne, J. Westerweel, F. Nieuwstadt, H. Faisst, B. Eckhardt, H. Wedin, R. Kerswell, and F. Waleffe, Science 305, 1594 (2004).

[22] B. Hof, A. Juel, and T. Mullin, Phys. Rev. Lett. 91, 244502 (2003).

[23] A. Draad, G. Kuiken, and F. Nieuwstadt, J. Fluid Mech. 377, 267 (1998).

[24] A. G. Darbyshire and T. Mullin, J. Fluid Mech. 289, 83 (1995).

[25] J. Hamilton, J. Kim, and F. Waleffe, J. Fluid. Mech. 287, 317 (1995).

[26] F. Waleffe, Phys. Fluids 9, 883 (1997).

[27] J. Jiménez and P. Moin, J. Fluid Mech. 225, 213 (1991).

[28] J. Jiménez and M. Simens, J. Fluid Mech. 435, 81 (2001).

[29] L. Brandt and D. Henningson, J. Fluid Mech. 472, 229 (2002).

[30] C. van Doorne, Ph.D. thesis, Delft University of Technology, 2004.

[31] A. K. Prasad, Exp. Fluids 29, 103 (2000).

[32] I. Wygnansky and F. Champagne, J. Fluid Mech. 59, 281 (1973). 